\title{
Review of: "Combining IL-6 and SARS-CoV-2 RNAaemia-based risk stratification for fatal outcomes of COVID-19"
}

\section{Yuan Shi}

Potential competing interests: The author(s) declared that no potential competing interests exist.

Interesting study combining IL-6 with covid-19 virus RNA and Oxygenation index to predict the clinical outcome. 